\title{
Some Quantum Operators with Discrete Spectrum but Classically Continuous Spectrum
}

\author{
BARRY SimON* \\ Departments of Mathematics and Physics, \\ California Institute of Technology, Pasadena, California 91125
}

Received September 22, 1982

\begin{abstract}
We consider a number of simple quantum Hamiltonians $H(-i \nabla, x)$ with the following property: $H(-i \nabla, x)$ has discrete spectrum even though $\{(p, q) \mid H(p, q)<E\}$ has infinite volume.
\end{abstract}

\section{INTRODUCTION}

A standard rule of thumb about whether a quantum Hamiltonian $H=-\Delta+V(x)$ has purely discrete spectrum or some continuous spectrum is the following. Look at the volume $\left\{(p, q) \mid p^{2}+V(q) \leqslant E\right\}$ in $R^{2 v}$. If this volume is finite for all $E$, the standard wisdom is that $H$ has only discrete spectrum. This standard wisdom also says that if this volume is infinite for some $E<\infty$, then the spectrum is not purely discrete. There are theorems which validate the first half of this intuition. Indeed, if $v \geqslant 3$, it is a consequence of the Cwickel-Lieb-Rosenbljum bound (see, e.g., [6] for references)

$$
N(E) \leqslant c_{v}\left|\left\{(p, q) \mid p^{2}+V(q) \leqslant E\right\}\right|,
$$

where $|\cdot| \equiv$ volume (Lebesgue measure) of $\cdot$ and $N(E)$ is the number of eigenvalues (counting multiplicity) of $H$ smaller than $E$.

Moreover, in any dimension, one has the Golden-Thompson inequality (see [6] again for details)

$$
Z_{Q}(t) \equiv \operatorname{Tr}\left(e^{-t H}\right) \leqslant Z_{\mathrm{cl}}(t)=(2 \pi)^{-v} \int d^{v} p d^{v} q e^{-t\left(p^{2}+V(q)\right)}
$$

and of course, if $Z_{Q}(t)<\infty$ for any $t$, then $H$ has purely discrete spectrum.

There are some indications that the other sides of the intuition, namely, $Z_{\mathrm{cl}}=\infty$ implies $Z_{Q}=\infty$, is correct. For example, in many cases [6], $Z_{\mathrm{cl}}(t) / Z_{Q}(t) \rightarrow 1$ as $t \downarrow 0$ so that one is tempted to believe this in general. The point of this article is to discuss

* Research partially supported by USNSF under Grant MCS-81-20833. 
a number of simple examples where $Z_{Q}(t)<\infty$ even though $\left|\left\{(p, x) \mid p^{2}+V(x) \leqslant E\right\}\right|$ is infinite.

The simplest example of this genre is the two dimensional Hamiltonian

$$
H_{1}=-\frac{\partial^{2}}{\partial x^{2}}-\frac{\partial^{2}}{\partial y^{2}}+x^{2} y^{2}
$$

indeed, my interest in these problems was first aroused when J. Goldstone and R. Jackiw asked me if $H_{1}$ had discrete spectrum. Closely related to this is the operator

$$
H_{2}=-\Delta_{D}^{\Omega} \quad \text { with zero boundary conditions on }\{(x, y)|| x y \mid \leqslant 1\} .
$$

Here the quantum/classical intuition and the consequence of $Z_{\mathrm{cl}} / Z_{Q} \rightarrow 1$ as $t \rightarrow \infty$ go back to a celebrated 70-year-old paper of Weyl. Since

$$
H_{2} \geqslant H_{1}-1
$$

any proof that $H_{1}$ has discrete spectrum will automatically imply that $H_{2}$ has discrete spectrum.

Both $H_{1}$ and $H_{2}$ have discrete spectrum with infinite classical phase space volumes. We will give five (!) proofs that $H_{2}$ has discrete spectrum; three work directly for $H_{1}$ also and the other two may well extend. Our reasons for giving so many proofs are several: Since the phenomenon is somewhat surprising it is worthwhile to give several proofs which provide distinct insights. More importantly, the proofs have distinct virtues: While the first is undoubtedly the "simplest," it gives a very bad bound on the high energy behavior of the eigenvalue distribution. For example, it shows $\operatorname{Tr}\left(e^{-t H_{1}}\right) \leqslant C t^{-3}$ for $t$ small, while the exact behavior is $O\left(t^{-3 / 2} \ln t\right)$.

In Section 2, we present the simple proof whose main ingredient is the zero point energy of the harmonic oscillator. Our second proof, in Section 3, uses boundary condition bracketing [5] and provides in an explicit way an explanation of the basic phenomena. The path integral proof of Section 4 is intermediate between that of Sctions 3 and 5. The proof in Section 5 depends on a new inequality whose proof we give elsewhere [7]. Its virtue is that it gives the exact leading behavior (including constants) as we explain in [7]. Section 6 deduces the required result from a general theorem of Fefferman and Phong [2], who became interested in similar problems because of some applications to PDEs. Since this proof is unpublished (although the ideas are almost identical to their proof in [1] of a bound on ground state energies) and since one can use Temple's inequality to simplify their proof, we give a proof of this result in an appendix. Their method gets the precise leading powers of divergence of $\operatorname{Tr}\left(e^{-t H}\right)$ as $t \downarrow 0$ but not the correct constant.

There is a sixth proof, whose details we spare the reader, but which we mention because it is connected with a precedent for these results. In two dimensions, let $x, y$ be the variables and $p, q$ the conjugate momentum. Let $\chi$ be the characteristic function of a compact region in $x-y$ space. Several years ago, Yajima [9] proved and used the fact that $\chi\left(p^{2}+q+i\right)^{-1}$ (note: $q$ not $q^{2}$ ) is compact. He noted, but did 
not publish, that the same ideas show that $\chi\left(p^{2}-q^{2}+i\right)^{-1}$ is compact. This somewhat surprising fact is clearly connected to the fact that $\left(H_{1}+1\right)^{-1}$ is compact and, indeed, one can prove the latter compactness from the former.

The biggest advantage of the Fefferman-Phong proof is that it extends quite simply to higher dimension (and provides necessary and sufficient conditions for $-\Delta+V$ to have discrete spectrum when $V$ is a polynomial). In the last two sections we describe two more interesting situations where their theorem is applicable. One is the model that motivated the question of Goldstone and Jackiw [3]. Let $a$ be a semisimple Lie algebra in compact form (i.e., the Killing form is strictly negative definite) and let $-\Delta$ be the Laplacian in the inner product given by the negative of the Killing form. Let $v \geqslant 2$ and let $a^{v}$ be $v$-tuples $\left(A_{1}, \ldots, A_{v}\right)$ of elements of $a$. Let $H$ be the operator on $L^{2}\left(a^{\nu}\right)$

$$
H=-\sum_{i} \Delta_{A_{i}}-\sum_{\mu<\kappa} \operatorname{Tr}\left(\left[A_{\mu}, A_{\kappa}\right]^{2}\right) .
$$

In Section 7, we will show this has purely discrete spectrum. This Hamiltonian has been proposed as a model of zero momentum Yang-Mills fields [3], but one that seems to be currently out of favor. In the last section, we consider three particles in three dimensions with their center of mass motion removed. The potential of the three particles is taken to be the area of the triangle formed by the three particles. Explicitly, if the particles have mass 1 and we use coordinates $x=r_{2}-r_{1}$, $y=r_{3}-\frac{1}{2}\left(r_{1}+r_{2}\right)$, then on $L^{2}\left(R^{6}\right)$

$$
H=-\Delta_{x}-\frac{3}{4} \Delta_{y}+|x \times y| .
$$

We will show in Section 8 that this operator (and others like it) have purely discrete spectrum.

Notice that operators (1), (3), (4) have a similar structure in common: Their potentials fail to go to infinity at infinity but only on very thin sets. This is the key to the breakdown of the classical intuition.

\section{First Proof: Zero Point Oscillator Motion}

It is, of course, well known that

$$
-\frac{d^{2}}{d q^{2}}+\omega^{2} q^{2} \geqslant|\omega| .
$$

Thus, treating $y$ as $c$-number,

$$
-\frac{d^{2}}{d x^{2}}+x^{2} y^{2} \geqslant|y|
$$


Using symmetry in $x$ and $y$ and adding, we see that

$$
\begin{aligned}
-\frac{d^{2}}{d x^{2}}-\frac{d^{2}}{d y^{2}}+x^{2} y^{2} & \geqslant \frac{1}{2}\left(-\frac{d}{d x^{2}}+x^{2} y^{2}\right)+\frac{1}{2}\left(-\frac{d}{d y^{2}}+x^{2} y^{2}\right)-\frac{1}{2} \Delta \\
& \geqslant \frac{1}{2}(-\Delta+|x|+|y|) \equiv H_{3} .
\end{aligned}
$$

Since $H_{3}$ has discrete spectrum, so does $H_{1}$. By the usual classical result [6], as $t \downarrow 0$

$$
\operatorname{Tr}\left(e^{-t H_{3}}\right)=[1+o(1)](2 \pi)^{-2} \int \exp \left(-t p^{2}-t|x|-t|y|\right) d x d y d^{2} p=c t^{-3}[1+o(1)]
$$

so (5) only yields

$$
\operatorname{Tr}\left(e^{-t H_{1}}\right) \leqslant c t^{-3}
$$

while the true behavior [7] is $O\left(t^{-3 / 2} \ln t\right)$, so (5) is not good at high energies (which is equivalent to small time): Explicitly, for $H_{1}, N(E)$ grows like $E^{3 / 2} \ln E$ while for $H_{3}$ it grows like $E^{3}$.

\section{Second Proof: Boundary Condition Bracketing}

We want to consider an array of rectangles as shown in Fig. 1, where dotted lines indicated Neumann $(N)$ lines, and solid lines are Dirichlet $(D)$ : Explicitly, the set has four-fold symmetry and the rectangles that intersect the positive $x$ axis are a square, $S_{0}$, with vertices at $( \pm 1, \pm 1)$ and $N$-sides, and rectangles $R_{n+t(x)}$ with vertices $\left(2^{n-1}\right.$, $\left.\pm 2^{-n+1}\right),\left(2^{n}, \pm 2^{-n+1}\right)$ and horizontal $D$-sides and vertical $N$-sides. Let $H_{4}$ denote the Laplacian in this sum of squares, and we claim

$$
H_{2} \geqslant H_{4} \text {. }
$$

For we go from $\mathrm{H}_{2}$ to $\mathrm{H}_{4}$ in two steps exploiting the properties of $D-N$ bracketing (e.g., $[4,5])$ : First we let $H_{3}$ be the Hamiltonian in the union of rectangles with DBC on all lines on the boundary of the union.

Then, as the region has been increased, $H_{2} \geqslant H_{5}$. Since adding $N$ lines or changing $D$ to $N$ lines always decreases $H, H_{5} \geqslant H_{4}$, so (6) is proven.

By $D-N$ decoupling, $H_{4}$ is the direct sum of Laplacians in each rectangle. $S_{0}$ yields an operator with eigenvalues $\left(\frac{1}{2} \pi\right)^{2}\left[m_{1}^{2}+m_{2}^{2}\right]\left(m_{i}=0,1, \ldots\right)$ and the four rectangles $R_{n, \pm y}^{x}$ yield eigenvalue $\pi^{2}\left[4^{(n-1)} k^{2}+4^{-(n-1)} m^{2}\right]$ with $m=0,1, \ldots$ and $k=1,2, \ldots$; since $k \geqslant 1$, one sees that $H_{4}$ has discrete spectrum (it has only finitely many eigenvalues smaller than a fixed $E$ ) and so, therefore, does $\mathrm{H}_{2}$.

This proof yields the correct power small $t$ dependence of $\operatorname{Tr}\left(e^{-t H_{2}}\right)$ but not the correct constant. 


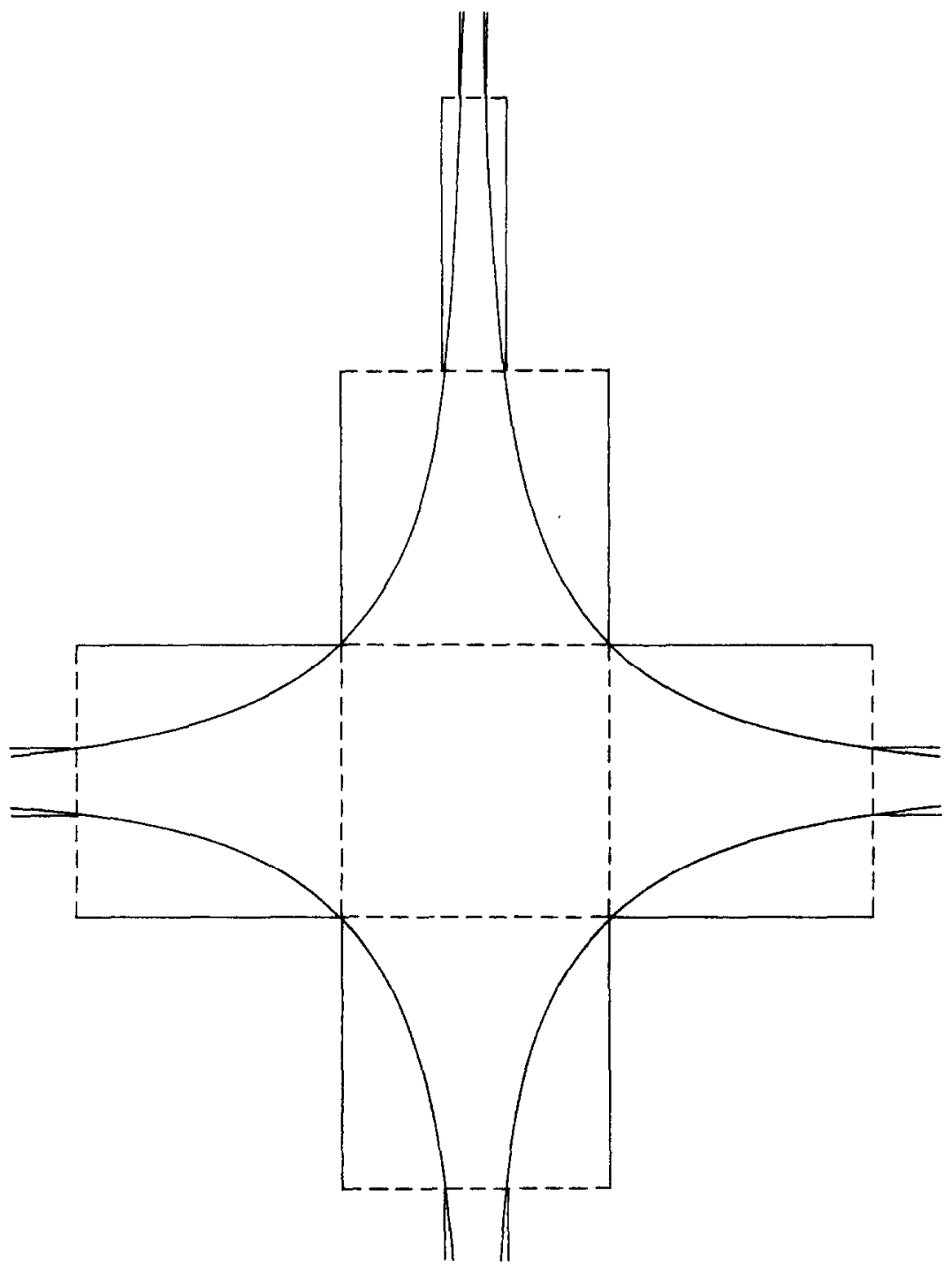

FIGURE 1

4. Third Proof: Path Integrals

This proof is intermediate between that in the last section and the one in the next. Indeed, the proof of the exact leading asymptotics we will give in [7] uses both ideas 
from this section and the next. We will prove $\operatorname{Tr}\left(e^{-i H_{2}}\right)<\infty$, which implies $H_{2}$ has discrete spectrum. By the Feynman-Kac formula (see, e.g., [6]),

$$
\begin{aligned}
\operatorname{Tr}\left(e^{-1 / 2 t H_{2}}\right) & =\int_{|x y| \leqslant 1} F(\vec{r}) d^{2} r, \\
F(\vec{r}) & =E_{\vec{r}, \vec{r}, t}\left(\chi_{\vec{r}}(b)\right)(2 \pi t)^{-1},
\end{aligned}
$$

where $E_{\vec{r}, \vec{r}, t}$ is Brownian motion expectation for all paths starting at $\vec{r}$ and conditioned to return to $\vec{r}$ at time $t$, and where $\chi_{\vec{r}}(b)$ is the characteristic function of all paths which stay in the region $|x y| \leqslant 1$. Let $r=(x, y)$ with $x>y$ and, say, $x>2$ and $|x y| \leqslant 1$. If $\left|b_{1}(s) b_{2}(s)\right| \leqslant 1$ for all $s$, then either $\left|b_{1}\right| \leqslant x / 2$ for some $s$ or else $\left|b_{2}(s)\right| \leqslant 2 / x$ for all $s$. By standard estimates (see [6]), the probability of the first event is bounded for large $x$ as $O\left(e^{-D x^{2} / t}\right)$ and by simple Dirichlet arguments, the second probability is $O\left(e^{-D^{\prime} x^{2} t}\right)$. Thus $F(\vec{r})$ behaves for $\vec{r}$ large as $O\left[\exp \left(-C r^{2}\right)\right]$ and thus $\operatorname{Tr}\left(e^{-t H_{2}}\right)<\infty$.

This proof easily yields the correct leading behavior as $t \downarrow 0$. By replacing $\left|b_{1}\right| \leqslant x / 2$ by $\left|b_{1}-x\right| \leqslant 1$ and doing the second estimate more carefully, one can even get the correct constants [7].

\section{Fourth Proof: Sliced Bread}

In [7], we will prove a general form of the following:

THEOREM 1 ("Sliced bread inequalities"). Let $V(x, y)$ be a function of two variables and let $H=-\Delta_{(x, y)}+V$. Let $h_{x}=-\left(d^{2} / d y^{2}\right)+V(x, y)$ as an operator on $\mathscr{H}_{y}=L^{2}(R, d y)$. Let $\varepsilon_{j}(x)$ be the eigenvalues of $h_{x}$ ordered by $\varepsilon_{1} \leqslant \varepsilon_{2} \leqslant \cdots$. Then

$$
\operatorname{Tr}\left(e^{-t H}\right) \leqslant \sum_{j} \operatorname{Tr}_{\mathscr{H}_{x}}\left[\exp \left\{-t\left(-\frac{d}{d x^{2}}+\varepsilon_{j}(x)\right)\right\}\right] .
$$

This result is easy to apply to see that $H_{1}$ has discrete spectrum. In that case, $\varepsilon_{j}(x)=(2 j+1)|x|$. Next, by scaling, $\left(\left(-d / d x^{2}\right)+\alpha|x|\right)$ is unitarily equivalent to $\alpha^{2 / 3}\left(\left(-d^{2} / d x^{2}\right)+|x|\right)$. Thus, if $F(s)=\operatorname{Tr}\left(\exp \left[-s\left(\left(-d^{2} / d x^{2}\right)+V\right)\right]\right)$, then (8) implies that

$$
\operatorname{Tr}\left(e^{-t H_{1}}\right) \leqslant \sum_{j=0}^{\infty} F\left((2 j+1)^{2 / 3} t\right)
$$

Since $F(s)$ decays exponentially as $s \rightarrow \infty$, the sum is finite and so $\operatorname{Tr}\left(e^{-t H}\right)<\infty$.

The small $s$ divergence of $F$ is determined by classical phase space [6] and from that one can read off the small $t$ divergence of the right side of (9). It has the same leading behavior, both power and constant as $\operatorname{Tr}\left(e^{-t H_{1}}\right)$ as can be seen by suitable lower bounds (see [7]). 


\section{Fifth Proof: The Fefferman-Phong Theorem}

Fefferman and Phong [2] have prove the following beautiful theorem:

TheOREm 2. Let $\Delta_{j}^{\lambda}\left(\lambda>0, j \in Z^{\nu}\right)$ be the cube of side $\lambda^{-1 / 2}$ centered at the point $\lambda^{-1 / 2} j$. Given $V \geqslant 0$ on $R^{v}$, let $\tilde{N}(\lambda)$ be the number of cubes $\Delta_{j}^{\lambda}$ with $\max _{x \in \Delta_{j}^{\lambda}} V(x) \leqslant \lambda$. Let $N(\lambda)$ be the dimension of the spectral projection for $-\Delta+V$ on the interval $(0, \lambda)$. Then, if $V$ is a polynomial of degree $d$ on $R^{v}$,

$$
\tilde{N}(b \lambda) \leqslant N(\lambda) \leqslant \tilde{N}(a \lambda)
$$

for all $\lambda$ and suitable constants $a, b$, where $b$ only depends on $v$ and $a$ depends on $v$ and the degree $d$.

Remarks. 1. While this theorem is unpublished, a very similar result appears for the ground state energy in [1]. The same proof extends to get this theorem. For the reader's convenience and because our proof of the crucial upper bound is partly alternative, we describe the proof in an appendix.

2. The lower bound is not very surprising. Indeed, it holds for any $V \geqslant 0$. The upper bound is much subtler. By taking $V$ to be a sum of narrower and higher spikes centered at the points $j 2^{-n}$ we can construct a $V$ with $\tilde{N}(\lambda)<\infty$ for all $\lambda$, but with $\sigma(H)=[0, \infty)$ so $N(\lambda) \equiv \infty$ !

3. As the example in 2 shows, one needs some regularity of $V$ to get the upper bound. Polynomial is not critical; rather, a glance at the proof shows that one needs the following: Given a function on $R^{n}$ not vanishing on any open set, let $G(f)$ be the function on the unit cube equal to $f(x) /\|f\|_{\infty}$, where $\|f\|_{\infty}=\sup _{x \in \Delta}|f(x)|$. Given $V$, and $\mu \in R^{+}, a \in R^{v}$ let $V_{\mu, a}(x)=V(\mu(x-a))$. Then the critical property is that $\left\{G\left(V_{\mu, a}\right) \mid \mu>0, a \in R^{v}\right\}$ has compact closure in $\|\cdot\|_{\infty}$. If $V$ is a polynomial of degree $d$ so is $V_{\mu, a}$ and thus $\left\{G\left(V_{\mu, a}\right)\right\}$ is the unit sphere in a finite dimensional space. Another case which is important in Section 8 is when $V$ is a (fractional) power of a polynomial. We use this extension without further comment.

4. The upper bound is also the more significant, since it says that $N(\lambda)<\infty$; i.e., $H$ has purely discrete spectrum if $\tilde{N}(\lambda)<\infty$ for all $\lambda$. (Indeed, $\tilde{N}(\lambda)<\infty$ for all $\lambda$ is equivalent to discreteness by the lower bound.) If $\tilde{N}(\lambda)$ (or $N(\lambda)$ ) has pure power (or power times logs) leader behavior, then $N(\lambda)$ must have the same leading behavior as $\tilde{N}(\lambda)$ although the constants will not be right. In practical situations, it may be easier to prove $\tilde{N}<\infty$ than to compute its asymptotics.

5. It is a reasonable conjecture that if $V$ is a positive polynomial, then $\tilde{N}(\lambda)<\infty$ unless $V$ is independent of some variable (in which case $\tilde{N}(\lambda)$ is $\infty$ for all $\lambda>0)$.

6. $\lambda^{-1 / 2}$ cubes enter since their kinetic energies are of order $\lambda$. 
It is easy to see that when $V(x, y)=x^{2} y^{2}$, then $\tilde{N}(\lambda)<\infty$. Indeed, if $j=\left(j_{1}, j_{2}\right)$

$$
\max _{\Delta \Delta_{j}^{\lambda}} V(x)=\lambda^{-2}\left(\left|j_{1}\right|+\frac{1}{2}\right)^{2}\left(\left|j_{2}\right|+\frac{1}{2}\right)^{2}
$$

so, without any effort, one sees that this maximum is larger than $\frac{1}{4} \lambda^{-2} \max \left(\left|j_{1}\right|,\left|j_{2}\right|\right)^{2}$ so $\tilde{N}(\lambda) \leqslant \lambda^{3}$. An only slightly more careful analysis yields the correct (up to constant $), \tilde{N}(\lambda) \leqslant c \lambda^{3 / 2} \ln \lambda$.

\section{A Lie Algebra Model}

In this section, we apply the Fefferman-Phong theorem to a special class of polynomials which will include the potential of Eq. (3).

THEOREM 3. Let $Q_{1}, \ldots, Q_{l}$ be real-valued, homogeneous polynomials of degree 2 on $R^{v}$ with

$$
\sum_{\substack{j=1, \ldots, l \\ \alpha=1, \ldots, \nu}}\left(\frac{\partial Q_{j}}{\partial x_{\alpha}}\right)^{2}>0
$$

when $x \neq 0$. Then the $\tilde{N}$ associated to

$$
P=\sum_{1}^{l} Q_{j}^{2}
$$

is finite for all $\lambda$.

Remarks 1 . The example $l=1, Q(x, y)=x y$ shows that $P$ can have zeros going out to infinity.

2. The fact that $\operatorname{deg} Q_{j}=2$ or even that all $Q_{j}$ have the same degree is irrelevant as is easy to see with slightly more argument, but the homogeneity is critical.

Proof. By scaling, it suffices to prove $\tilde{N}(\lambda=1)<\infty$. Obviously $S(x) \equiv \max _{j, \alpha}\left|\partial Q_{j} / \partial x_{\alpha}\right|>0$ if (11) holds. Since $S(\lambda x)=\lambda S(x)$ (by homogeneity of $Q)$, we see that

$$
\min _{|x|=R} S(x)=c R .
$$

Thus, for any box $\Delta_{k}$ we have that for some $j, a,\left|\partial Q_{j} / \partial x_{\alpha}\right| \geqslant c k$ at the center of $\Delta_{k}$. Since $\max _{\alpha, \beta, j}\left|\partial^{2} Q_{j} / \partial x_{\alpha} \partial x_{\beta}\right|=d<\infty,\left|\partial Q_{j} / \partial x_{\alpha}\right| \geqslant c k-(d / 2) \sqrt{v}$ in $\Delta_{k}$. Thus, if $k \geqslant \frac{1}{2} d c^{-1} \sqrt{v}$, and $x, x^{\prime}$ are suitable points in $\Delta_{k}$ ("opposite" in the $\alpha$ th direction), $\left|Q_{j}(x)-Q_{j}\left(x^{\prime}\right)\right| \geqslant c k-(d / 2) \sqrt{v}$. Thus, if $c k-(d / 2) \sqrt{v}>2, \max _{\Delta_{j}}\left|Q_{j}\right|>1$ and thus $\max _{\Delta_{j}} P>1$. Such a cube does not count in $\tilde{N}(1)$. Thus if $|j|$ is sufficiently large, $\Delta_{j}$ doesn't count in $\tilde{N}(1)$ and so $\tilde{N}(1)<\infty$. 
COROLlary 4. The operator of (3) has purely discrete spectrum.

Proof. The potential, $P$, is a polynomial, so by the Fefferman-Phong theorem, we need only check that $\tilde{N}(\lambda)<\infty$. P has the form of the last theorem, so we need only check that (11) holds. There is presumably a lengthy but elegant coordinate-less proof, but we settle for a direct calculation in coordinates. Pick a basis for the Lie algebra with $\operatorname{Tr}\left(E_{i} E_{j}\right)=-\delta_{i j}$ (recall the elements of the algebra are skew-symmetric). This basis may not be Killing orthonormal, but if (11) holds in any linear basis, it is easy to see it holds in all linear bases, so we can check in this basis.

As a preliminary, we write commutators in terms of structure constants:

$$
\left[E_{i}, E_{j}\right]=\sum_{k} c_{i j}^{k} E_{k} .
$$

Obvious $c_{i j}^{k}$ is antisymmetric under interchanges of $i$ and $j$, and since $c_{i j}^{k}=$ $-\operatorname{Tr}\left(\left[E_{i}, E_{j}\right] E_{k}\right)$ it is also seen to be antisymmetric under other interchanges. If $A=\sum a^{i} E_{i}$, then in $E_{i}$ basis

$$
(\operatorname{Ad} A)_{j}^{k}=\sum_{i} a^{i} c_{i j}^{k}
$$

is antisymmetric by the above remark, and thus the strict negative definiteness of the Killing form becomes

$$
\sum_{i, l . k, j} a^{i} c_{i j}^{k} a^{l} c_{l j}^{k}>0
$$

Now write $A_{\mu}=\sum a_{\mu}^{i} E_{i}$ so $\left\{a_{\mu}^{i}\right\}$ are the basic coordinates of the problem. The potential $P$ has the form (since $\left.-\operatorname{Tr}\left(E_{i}, E_{j}\right)=\delta_{i j}\right)$

where

$$
\begin{aligned}
P & \left.=-\sum_{\substack{\mu<\kappa \\
i, j, l, m}} a_{\mu}^{i} a_{\kappa}^{j} a_{\mu}^{l} a_{\kappa}^{m} \operatorname{Tr}\left(\left[E_{i}, E_{j}\right] \mid E_{l}, E_{m}\right]\right) \\
& =\sum_{\substack{\mu, \kappa \\
i, j, k, l, m}} a_{\mu}^{i} a_{\kappa}^{j} a_{\mu}^{l} a_{\kappa}^{m} c_{i j}^{k} c_{l m}^{k} \\
& =\sum_{k, \mu, k} Q_{\mu, \kappa}^{k}(a),
\end{aligned}
$$

$$
Q_{\mu, \kappa}^{k}(a)=\sum_{i, j} a_{\mu}^{i} a_{\kappa}^{j} c_{i j}^{k}
$$

For any $\left\{A^{\mu}\right\} \neq\{0\}$, pick $\mu$ with $A^{\mu} \neq 0$ and any $\kappa$. Then (13) says that $\sum_{\kappa, j}\left(\partial Q_{\mu, \kappa}^{k} / \partial a_{\kappa}^{j}\right)^{2}>0$ and thus (11) holds.

Remark. The proof works for any faithful representation of $a$ by skew-symmetric matrices. 


\section{A Model of Feynman}

The Hamiltonian (4) was proposed by Feynman as a pedagogical problem. $\mathrm{He}$ regards it as obvious that the spectrum is discrete, but it is perhaps worth giving a rigorous proof of what Feynman regards as obvious! To handle higher dimension than 3 (or dimension 2), we let $\mu \geqslant 2$ and $v=2 \mu$. We write a point in $R^{v}$ as $(x, y)$ with $x, y \in R^{v}$ and let

$$
H=-\Delta_{x}-\Delta_{y}+c\left[\sum_{i<j}\left(x_{i} y_{j}-x_{j} y_{i}\right)^{2}\right]^{1 / 2},
$$

where $c>0$. By scaling (4) is a special case of (14).

THEOREM 5. The operator of (14) has purely discrete spectrum.

Proof. By the Fefferman-Phong theorem (in the extended form mentioned in Remark 3 of Section ), we only need show $\tilde{N}(\lambda)<\infty$. The proof of Theorem 3 is seen to extend to $V=\left(\sum Q_{j}^{2}\right)^{1 / 2}$, so we let $Q_{i j}(x, y)=x_{i} y_{j}-x_{j} y_{i}$. It suffices that $\sum_{i, j, k}\left(\partial Q_{i j} / \partial x_{k}\right)^{2}+\left(\partial Q_{i j} / \partial y_{k}\right)^{2} \equiv G(x)$ not vanish away from zero. But by an immediate calculation

$$
G(x)=2(\mu-1)\left[x^{2}+y^{2}\right]
$$

and the result follows.

Remark. By a simple extension of the argument, if $k+1$ particles living in dimension $\mu \geqslant k$ interact by a potential which is equal to the $k$-form "volume" spanned by the $k-1$ particles, then the spectrum is purely discrete.

\section{APPENDIX: Proof of the Fefferman-Phong Theorem}

Our goal here is to prove Theorem 2 in a series of lemmas. The two inequalities appear as Lemmas A.1 and A.5. As a preliminary, we exploit Dirichlet-Neuman bracketing which assures us that

$$
N_{D}(\lambda) \leqslant N(\lambda) \leqslant N_{N}(\lambda),
$$

where $N_{X}(\lambda)$ is the dimension of the spectral projection on $[0, \lambda]$ for the operator $H_{X}(\lambda)$, with $X$-boundary conditions on all boxes $\left\{\boldsymbol{A}_{j}^{\lambda}\right\}_{j \in Z^{*}}$.

LEMmA A.1. $\quad N\left(\left(1+v \pi^{2}\right) \lambda\right) \geqslant \tilde{N}(\lambda)$.

Proof. If $\Delta_{j}^{\lambda}$ is a $\lambda$-box with $\max _{\Delta_{j}} V \leqslant \lambda$, then $H_{0}^{D}+V$ has lowest eigenvalue at most $v \lambda \pi^{2}+\lambda$, so there are at least $\tilde{N}(\lambda)$ eigenvalues of $H_{D}(\lambda)$ of size at most $\left(1+v \pi^{2}\right) \lambda$. 
The following standard result of Temple [8] is provided for the reader's convenience:

Lemma A.2 (Temple's inequality). Let $A$ be a selfadjoint operator whose two lowest eigenvalues are $\mu_{1}<\mu_{2}$. Suppose that $\varphi$ is a unit trial vector in $D(A)$ and $\mu_{2}^{*}$ a number with

$$
(\varphi, A \varphi)<\mu_{2}^{*} \leqslant \mu_{2} .
$$

Then

$$
\mu_{1} \geqslant(\varphi, A \varphi)-\left[\mu_{2}^{*}-(\varphi, A \varphi)\right]^{-1}\left\{\left(\varphi, A^{2} \varphi\right)-(\varphi, A \varphi)^{2}\right\} .
$$

Proof. $\left(A-\mu_{2}^{*}\right)\left(A-\mu_{1}\right) \geqslant 0$ since $\left(A-\mu_{2}^{*}\right)$ is only non-positive on the $\mu_{1}$ eigenspace and $\left(A-\mu_{1}\right)$ kills that space. Thus $\left(\varphi,\left(A-\mu_{2}^{*}\right)\left(A-\mu_{1}\right) \varphi\right) \geqslant 0$. This and (A.2) yield (A.3).

Lemma A.3. Fix $v, d$. Then there is a constant $c(v, d)$ so that for all polynomials $P$ on $R^{v}$ of degree at most $d$ and all cubes, $\Delta$ in $R^{v}$, we have that

$$
\frac{1}{\Delta} \int_{\Delta}|P(x)| d x \geqslant c \max _{\Delta}|P(x)|
$$

Proof (essential idea in [1]). By scaling and translating $x$ and scaling $P$, we can suppose that $\Delta$ is the unit cube centered about 0 and that $\|P\|_{\infty} \equiv \max _{\Delta}|P(x)|=1$. The set of $P$ is clearly compact since it is a unit sphere in a finite dimensional vector space and $\int_{\Delta}|P(x)| d x$ is a non-vanishing continuous function on that set, so the minimum value, $c$, is strictly positive.

Lemma A.4. Let $\Delta_{0}$ be the unit cube in $R^{v}$. Let $V$ be a positive polynomial of degree at most $d$ and let $H=H_{0, N}+V$, where $H_{0, N}$ is the Neuman Laplacian on $\Delta_{0}$. Suppose that $\max _{\Delta_{0}} V(x) \geqslant 1$. Then

$$
H \geqslant g \equiv c\left[\frac{\pi^{2}-2}{\pi^{2}-1}\right]
$$

where $c$ is given by (A.4).

Proof. By decreasing $V$ which only decreases $H$, we can suppose $\max _{\Delta_{0}} V=1$. Let $\mu_{1}, \mu_{2}$ be the two lowest eigenvalues of $H$. Since $H \geqslant H_{0, N}$, which has 0 and $\pi^{2}$ as its lowest eigenvalues, we see that $\mu_{2} \geqslant \mu_{2}^{*} \equiv \pi^{2}$. Let $\varphi$ be the vector which is identically 1. Then $\langle\varphi, H \varphi\rangle=\langle\varphi, V \varphi\rangle=\int_{\Delta} V(x) d x \leqslant 1\left\langle\mu_{2}^{*}\right.$. Moreover, $\left\langle\varphi, H^{2} \varphi\right\rangle-$ $\langle\varphi, H \varphi\rangle^{2} \leqslant\left\langle\varphi, H^{2} \varphi\right\rangle=\left\langle\varphi, V^{2} \varphi\right\rangle \leqslant\langle\varphi, V \varphi\rangle$ since $\max V=1$. Thus, by Temple's ine quality

$$
\begin{aligned}
\mu_{1} & \geqslant\langle\varphi, V \varphi\rangle\left[1-\left(\mu_{2}^{*}-1\right)^{-1}\right] \\
& \geqslant c\left(\pi^{2}-2\right) /\left(\pi^{2}-1\right) .
\end{aligned}
$$


LEMMA A.5. If $g$ is given by (A.5), then $N(g \lambda) \leqslant N(\tilde{\lambda})$.

Proof. Note that since $c<1$, we have that $g<1$. Any $\lambda$ box has a second eigenvalue at least $\lambda \pi^{2}>g \lambda$, so we get an upper bound on $N_{N}(g \lambda)$ by counting boxes for which $N_{0, N}+V$ has its lowest eigenvalues smaller than $g \lambda$. By scaling and Lemma A.4, if $\max _{\Delta \hat{j}} V(x)>\lambda$, this box does not contribute so $N_{N}(g \lambda) \leqslant N(\tilde{\lambda})$.

\section{ACKNOWLEDGMENTS}

It is a pleasure to thank J. Goldstone and R. Jackiw for raising their question, C. Fefferman for telling me of his work with D. Phong, and M. Aizenman, C. Fefferman, M. Peskin, and most especially J. Avron, for valuable discussions.

Note added in proof. In a 1948 paper (Das Eigenwertproblem von $\Delta u+\lambda u=0$ in Halbröhren, pp. 329-344 in "Studies and Essays" (K. Friedrichs, O. Neugebauer, and J. Stoker, eds.) Interscience, New York, 1948), F. Rellich obtained results closely related to those in this paper. There he proves certain infinite volume regions have Dirichlet Laplacians with discrete spectrum. Since he assumes his regions $G$ are "half-tubes" in the sense that in $R^{\nu},\left\{x \in G \mid x_{v}<a\right\}$ is bounded for each $a$, his results do not technically include those in this paper. However, his method, based on "Rellich compactness criteria" which is vaguely connected to our second proof, easily extends to the following general result: Given $V\left(x_{1}, \ldots, x_{v}\right)$ (allowed to be infinite), bounded below, define $E_{j}(a)$ to be the lowest eigenvalue of the operator $-\sum_{l \neq j}\left(\partial^{2} / \partial x_{i}^{2}\right)+V\left(x_{j}-a\right)$ on $L^{2}\left(x_{1}, \ldots, x_{j-1}, x_{j+1}, \ldots, x_{v}\right)$. If, for each $j, \lim _{|a| \rightarrow \infty} E_{j}(a)=\infty$, then $-\Delta+V$ has discrete spectrum. This general result easily implies all our two-dimensional results and perhaps, with some geometry, our other results. I would like to thank J. Goldstone and R. Jackiw for bringing this paper to my attention, and Alan Sokal for getting me a copy of it.

\section{REFERENCES}

1. C. Fefferman and D. Phong, Commun. Pure Appl. Math. 34 (1981), 285-331.

2. C. Fefferman and D. Phong, unpublished.

3. J. GoldSTONE AND R. JACKIW, private communication.

4. M. ReEd AND B. Simon, "Methods of Modern Mathematical Physics, IV. Analysis of Operators," Academic Press, New York, 1978.

5. B. Simon, $A d v$. Math. 30 (1978), 268-281.

6. B. Simon, "Functional Integration and Quantum Physics," Academic Press, New York, 1979.

7. B. Simon, J. Func. Anal., to appear.

8. G. Temple, Proc. Roy. Soc. A 119 (1928), 276-293.

9. K. Yajima, J. Math. Soc. Japan 29 (1977), 729-743. 